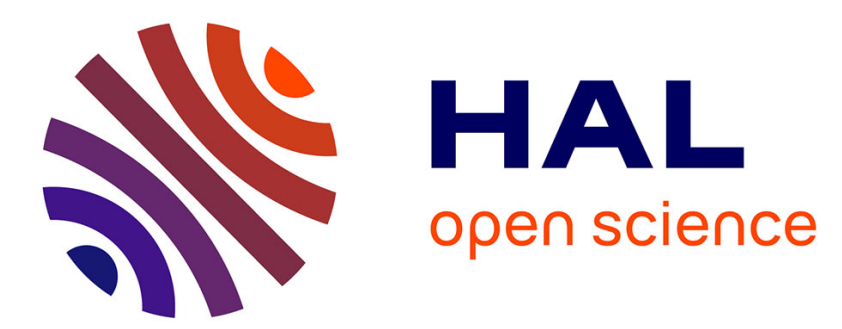

\title{
Expressiveness of Full First Order Constraints in the Algebra of Finite or Infinite Trees
}

\author{
Alain Colmerauer, Thi-Bich-Hanh Dao
}

\section{To cite this version:}

Alain Colmerauer, Thi-Bich-Hanh Dao. Expressiveness of Full First Order Constraints in the Algebra of Finite or Infinite Trees. Principles and Practice of Constraint Programming - CP 2000, 2000, Singapore, Singapore. pp.172-186. hal-00144924

\section{HAL Id: hal-00144924 \\ https://hal.science/hal-00144924}

Submitted on 25 Mar 2010

HAL is a multi-disciplinary open access archive for the deposit and dissemination of scientific research documents, whether they are published or not. The documents may come from teaching and research institutions in France or abroad, or from public or private research centers.
L'archive ouverte pluridisciplinaire HAL, est destinée au dépôt et à la diffusion de documents scientifiques de niveau recherche, publiés ou non, émanant des établissements d'enseignement et de recherche français ou étrangers, des laboratoires publics ou privés. 


\title{
Expressiveness of full first order constraints in the algebra of finite or infinite trees
}

\author{
Alain Colmerauer and Thi-Bich-Hanh Dao \\ Laboratoire d'Informatique de Marseille, CNRS, \\ Universités de la Méditerranée et de Provence
}

\begin{abstract}
We are interested in the expressiveness of constraints represented by general first order formulae, with equality as unique relational symbol and functional symbols taken from an infinite set $F$. The chosen domain is the set of trees whose nodes, in possibly infinite number, are labeled by elements of $F$. The operation linked to each element $f$ of $F$ is the mapping $\left(a_{1}, \ldots, a_{n}\right) \mapsto b$, where $b$ is the tree whose initial node is labeled $f$ and whose sequence of daughters is $a_{1}, \ldots, a_{n}$.

We first consider constraints involving long alternated sequences of quantifiers $\exists \forall \exists \forall \ldots$. We show how to express winning positions of two-partners games with such constraints and apply our results to two examples.

We then construct a family of strongly expressive constraints, inspired by a constructive proof of a complexity result by Pawel Mielniczuk. This family involves the huge number $\alpha(k)$, obtained by evaluating top down a power tower of 2's, of height $k$. With elements of this family, of sizes at most proportional to $k$, we define a finite tree having $\alpha(k)$ nodes, and we express the result of a Prolog machine executing at most $\alpha(k)$ instructions.

By replacing the Prolog machine by a Turing machine we rediscover the following result of Sergei Vorobyov: the complexity of an algorithm, deciding whether a constraint without free variables is true, cannot be bounded above by a function obtained by finite composition of elementary functions including exponentiation.

Finally, taking advantage of the fact that we have at our disposal an algorithm for solving such constraints in all their generality, we produce a set of benchmarks for separating feasible examples from purely speculative ones. Among others we solve constraints involving alternated sequences of more than 160 quantifiers.
\end{abstract}

\section{Introduction}

The algebra of (possibly) infinite trees plays a fundamental act in computer science: it is a model for data structures, program schemes and program executions. As early as 1976, Gérard Huet proposed an algorithm for unifying infinite terms, that is solving equations in that algebra [11]. Bruno Courcelle has studied the properties of infinite trees in the scope of recursive program schemes $[8,9]$. Alain Colmerauer has described the execution of Prolog II, III and IV programs in terms of solving equations and disequations in that algebra [4-6,1]. Michael 
Maher has introduced and justified a complete theory of the algebra of infinite trees [12]. Among others, he has shown that in this theory, and thus in the algebra of infinite trees, any first order formula is equivalent to a Boolean combination of conjunctions of equations (partially or totally) existentially quantified. Sergei Vorobyov has shown that the complexity of an algorithm, deciding whether a formula without free variables is true in that theory, cannot be bounded above, by a function obtained by finite composition of elementary functions, including exponentiation [14]. Pawel Mielniczuk has shown a similar result in the theory of feature trees, but with a more constructive method, which has inspired some of our examples [13].

We have recently developed an algorithm for solving general first order constraints in the algebra of infinite trees [10]. The purpose of this paper is not the presentation of this algorithm, but of examples, first imagined as tests, then extended to show the expressiveness of such general constrains. The paper is organized as follows.

(1) We end this first section by making clear the notions of tree algebra and first order constraints in that algebra.

(2) In the second section we consider constraints involving long alternated sequences of quantifiers $\exists \forall \exists \forall \ldots$. We show how to express winning positions of two-partners games with such constraints and apply our results to two examples.

(3) In the third section, we investigate the most expressive family of constraints we know. It involves the truly huge number $\alpha(k)$, obtained by evaluating top down a tower of powers of 2's, of height $k$. With elements of this family, of sizes at most proportional to $k$, we define a finite tree having $\alpha(k)$ nodes, and we express the result of a Prolog machine executing at most $\alpha(k)$. By replacing the Prolog machine by a Turing machine we rediscover the complexity result of Sergei Vorobyov mentioned at the beginning of this section. This part has been strongly influenced by the work of Pawel Mielniczuk [13].

(4) We conclude by discussions and benchmarks separating the feasible examples from the purely speculative ones.

\subsection{The algebra of infinite trees}

Trees are well known objects in the computer science world. Here are some of them:
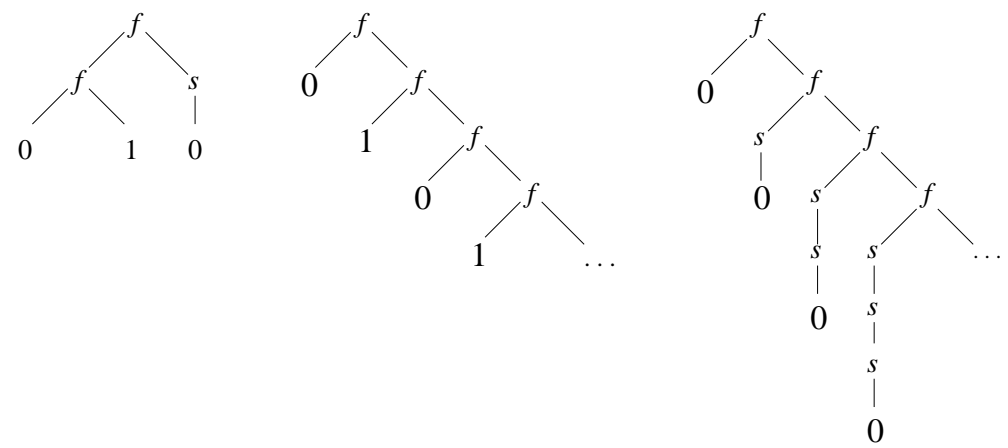
Their nodes are labeled by the symbols $0,1, s, f$, of respective arities $0,0,1,2$, taken from a set $F$ of functional symbols, which we assume to be infinite. Note that the first tree is the only one having a finite set of nodes, but that the second one has still a finite set of (patterns of) subtrees. We denote by $\mathbf{A}$ the set of all trees ${ }^{1}$ constructed on $F$.

We introduce in $\mathbf{A}$ a set of construction operations ${ }^{2}$, one for each element $f \in F$ which is the mappings $\left(a_{1}, \ldots, a_{n}\right) \mapsto b$, where $n$ is the arity of $f$ and $b$ the tree whose initial node is labeled $f$ and the sequence of daughters is $\left(a_{1}, \ldots, a_{n}\right)$ and which be schematized as

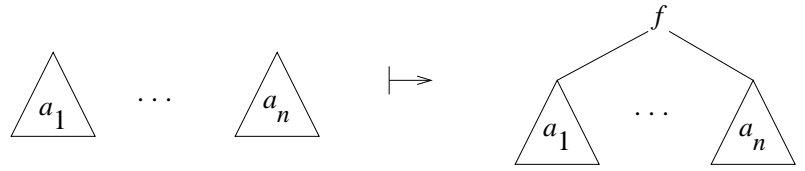

We thus obtain the algebra of infinite trees constructed on $F$, which we denote by $(\mathbf{A}, F)$.

\subsection{Tree constraints}

We are interested in the expressiveness of constraints represented by general first order formulae, with equality as unique relational symbol and functional symbols taken from an infinite set $F$. These tree constraints are of one of the 9 forms:

$$
s=t, \text { true, false, } \neg(p),(p \wedge q),(p \vee q),(p \rightarrow q), \exists x p, \forall x p,
$$

where $p$ and $q$ are shorter tree constraints, $x$ a variable taken from an infinite set and $s, t$ terms, that are expressions of one of the forms

$$
x, f t_{1} \ldots t_{n}
$$

where $n \geq 0, f \in F$, with arity $n$, and the $t_{i}$ 's are shorter terms.

The variables represent elements of the set $\mathbf{A}$ of trees constructed on $F$ and the functional symbols $f$ are interpreted as construction operations in the algebra of infinite trees $(\mathbf{A}, F)$. Thus a constraint without free variables is either true or false and a constraint $p\left(x_{1}, \ldots, x_{n}\right)$ with $n$ free variables $x_{i}$ establish an $n$-ary relation in the set of trees.

\footnotetext{
${ }^{1}$ More precisely we define first a node to be a word constructed on the set of strictly positive integers. A tree $a$, constructed on $F$, is then a mapping of type $E \rightarrow F$, where $E$ is a non-empty set of nodes, each one $i_{1} \ldots i_{k}$ (with $k \geq 0$ ) satisfying the two conditions: (1) if $k>0$ then $i_{1} \ldots i_{k-1} \in E$, (2) if the arity of $a\left(i_{1} \ldots i_{k}\right)$ is $n$, then the set of nodes of $E$ of the form $i_{1} \ldots i_{k} i_{k+1}$ is obtained by giving to $i_{k+1}$ the values $1, \ldots, n$.

${ }^{2}$ In fact, the construction operation linked to the $n$-ary symbol $f$ of $F$ is the mapping $\left(a_{1}, \ldots, a_{n}\right) \mapsto b$, where the $a_{i}$ 's are any trees and $b$ is the tree defined as follows from the $a_{i}$ 's and their set of nodes $E_{i}$ 's: the set $E$ of nodes of $a$ is $\{\varepsilon\} \cup\{i x \mid x \in$ $E_{i}$ and $\left.i \in 1 . . n\right\}$ and, for each $x \in E$, if $x=\varepsilon$, then $a(x)=f$ and if $x$ is of the form $i y$, with $i$ being an integer, $a(x)=a_{i}(y)$.
} 


\section{Long nesting of alternated quantifiers}

We first introduce the notions of $k$-winning and $k$-losing position in any twopartners games and in two examples. We show how to express, in any domain, the set of $k$-winning positions by a constraint. We end the section by expressing the $k$-winning positions of the two examples by tree constraints involving an alternated embedding of $2 k$ quantifiers.

\subsection{Winning positions in a two-partners game}

Let $(V, E)$ be a directed graph, with $V$ a set of vertices and $E \subseteq V \times V$ a set of edges. The sets $V$ and $E$ may be empty and the elements of $V$ are also called positions. We consider a two-partners game which, given an initial position $x_{0}$, consists, one after another, in choosing a position $x_{1}$ such that $\left(x_{0}, x_{1}\right) \in E$, then a position $x_{2}$ such that $\left(x_{1}, x_{2}\right) \in E$, then a position $x_{3}$ such that $\left(x_{2}, x_{3}\right) \in E$ and so on... The first one who cannot play any more has lost and the other one has won. For example the two following infinite graphs correspond to the two following games:
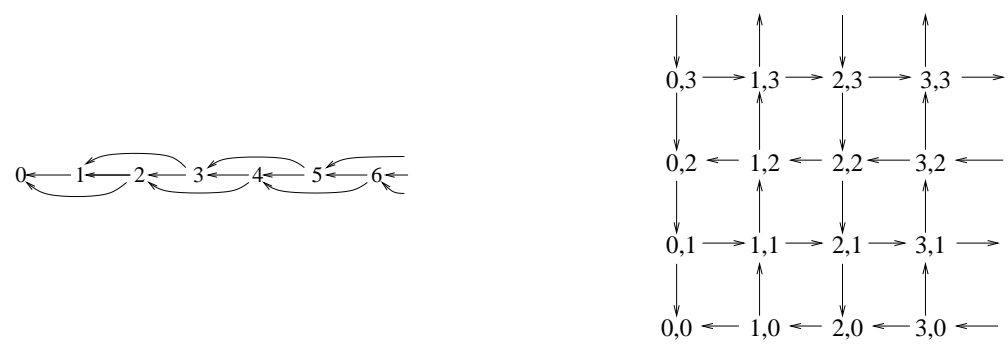

Game 1 A non-negative in- Game 2 An ordered pair $(i, j)$ of non-negative teger $i$ is given and, one after integers is given and, one after another, each another, each partner sub- partner chooses one of the integers $i, j$. Dependtracts 1 or 2 from $i$, but keep- ing on the fact that the chosen integer $u$ is odd ing $i$ non-negative. The first or even, he then increases or decreases the other person who cannot play any integer $v$ by 1 , but keeping $v$ non-negative. The more has lost. first person who cannot play any more has lost.

Let $x \in V$ be any vertex of the directed graph $(V, E)$ and suppose that it is the turn of person $A$ to play. The position $x$ is said to be $k$-winning if, no matter the way the other person $B$ plays, it is always possible for $A$ to win in making at most $k$ moves. The position $x$ is said to be $k$-losing if, no matter the way $A$ plays, $B$ can always force $A$ to lose and to play at most $k$ moves.

Consider the two preceding graphs and mark with $+k$ the positions which are $k$-winning and with $-k$ the positions which are $k$-losing, with each time $k$ being as small as possible. Vertex 0 of the first graph and vertex $(0,0)$ of the second one being the only 0 -losing positions, are marked with -0 . Starting from 
the vertices marked with -0 and following the arrows in reverse direction, we find successively the set of vertices to be marked by +1 , then -1 , then +2 , then -2 , then +3 , then -3 , and so on. We get
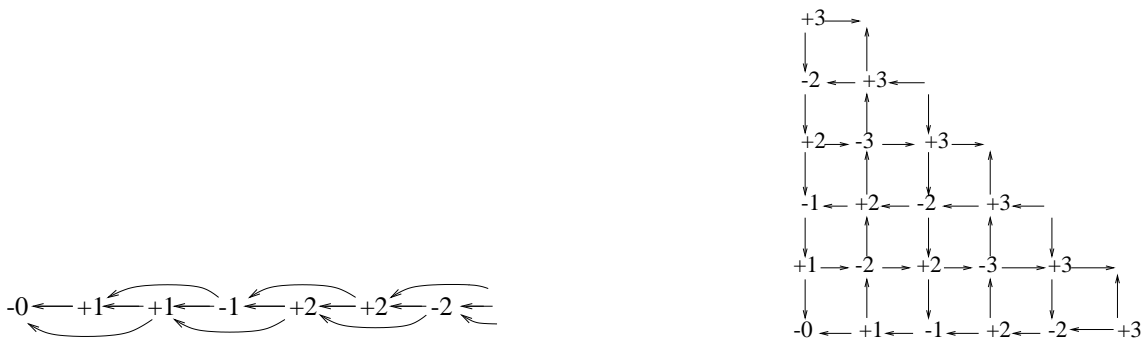

and convince ourselves that the set of $k$-winning positions of game 1 is

$$
\{i \in \mathbf{N} \mid i<3 k \text { and } i \bmod 3 \neq 0\}
$$

and of game 2

$$
\left.\{(i, j)) \in \mathbf{N}^{2} \mid i+j<2 k \text { and }(i+j) \bmod 2=1\right\} .
$$

where $\mathbf{N}$ is the set of non-negative integers.

\subsection{Expressing $k$-winning positions by a constraint}

Let $\mathbf{D}$ be a domain, that is a non-empty set and let $G=(V, E)$ the graph of a two-partners game, with $V \subseteq \mathbf{D}$. We will express the $k$-winning positions of $G$ by a constraint in $\mathbf{D}$ involving an embedding $\exists \forall \exists \ldots$ of $2 k$ alternated quantifiers.

Let us introduce in $\mathbf{D}$ the properties move, winning ${ }_{k}$ et $\operatorname{losing}_{k}$, defined by

$$
\begin{array}{ll}
\operatorname{move}(x, y) & \leftrightarrow(x, y) \in E, \\
\text { winning }_{k}(x) & \leftrightarrow x \text { is a } k \text {-winning position of } G, \\
\operatorname{losing}_{k}(x) & \leftrightarrow x \text { is a } k \text {-losing position of } G .
\end{array}
$$

In $\mathbf{D}$ we then have the equivalences, for all $k \geq 0$ :

$$
\begin{array}{ll}
\text { winning }_{0}(x) & \leftrightarrow \text { false, } \\
\text { winning }_{k+1}(x) & \leftrightarrow \exists y \text { move }(x, y) \wedge \operatorname{losing}_{k}(y), \\
\operatorname{losing}_{k}(x) & \leftrightarrow \forall y \operatorname{move}(x, y) \rightarrow \text { winning }_{k}(y) .
\end{array}
$$

Contrary to what we may believe, it follows that we have:

$$
\text { winning }_{k}(x) \rightarrow \text { winning }_{k+1}(x), \quad \operatorname{losing}_{k}(x) \rightarrow \operatorname{losing}_{k+1}(x) .
$$

Indeed, from the first and the last equivalence of (2) we conclude that these implications hold for $k=0$ and, if we assume that they hold for a certain $k \geq 0$, from the last two equivalences in (2) we conclude that they also hold for $k+1$. 


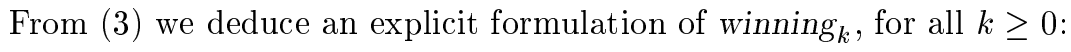

$$
\text { winning }_{k}(x) \leftrightarrow\left[\begin{array}{l}
\exists y \operatorname{move}(x, y) \wedge \neg( \\
\exists x \operatorname{move}(y, x) \wedge \neg( \\
\exists y \operatorname{move}(x, y) \wedge \neg( \\
\exists x \operatorname{move}(y, x) \wedge \neg( \\
\ldots \\
\exists y \operatorname{move}(x, y) \wedge \neg( \\
\exists x \operatorname{move}(y, x) \wedge \neg( \\
\text { false } \underbrace{) \ldots}_{2 k}
\end{array}\right]
$$

where of course all the quantifiers apply on elements of $\mathbf{D}$. By moving down the negations, we thus get an embedding of $2 k$ alternated quantifiers.

In equivalence (3) it is possible to use a more general definition of move than the one given in (1). We first remark, that for any non-negative $k$, the following property holds:

Property 1 Let three directed graphs be of the form $G_{1}=\left(V_{1}, E_{1}\right), G_{2}=$ $\left(V_{2}, E_{2}\right)$ and $G=\left(V_{1} \cup V_{2}, E_{1} \cup E_{2}\right)$. The graphs $G_{1}$ and $G$ have the same set of $k$-winning positions, if both:

1. the sets of vertices $V_{1}$ and $V_{2}$ are disjoint,

2. for all $x \in V_{2}$, there exists $y \in V_{2}$ with $(x, y) \in E_{2}$.

Indeed, from the first condition it follows that $E_{1}$ and $E_{2}$ are disjoint and thus that the set of $k$-winning positions of $G$ is the union of the set of $k$-winning positions of $G_{1}$ with the set of $k$-winning positions of $G_{2}$. This last set is empty because of the second condition.

It follows that:

Property 2 (Generalized move relation) Equivalence (3) holds also for any move relation obeying to the two conditions:

1. for all $x \in V$ and $y \in V$, move $(x, y) \leftrightarrow(x, y) \in E$,

2. for all $x \in \boldsymbol{D}-V$ there exists $y \in \boldsymbol{D}-V$ such that move $(x, y)$.

\subsection{Formalizing game 1 in the algebra of infinite trees}

We now reconsider game 1 introduced in section 2.1. As domain $\mathbf{D}$ we take the set $\mathbf{A}$ of trees constructed on a set $F$ of functional symbols including among others the symbols $0, s$, of respective arities 0,1 . We code the vertices $i$ of the game graph by the trees ${ }^{3} s^{i}(0)$. Let $G=(V, E)$ be the graph obtained this way.

As generalized relation move we then can take in the algebra of infinite trees:

$$
\operatorname{move}(x, y) \stackrel{\text { def }}{=} x=s(y) \vee x=s(s(y)) \vee(\neg(x=0) \wedge \neg(\exists u x=s(u)) \wedge x=y)
$$

${ }^{3}$ Of course, $s^{0}(0)=0$ and $s^{i+1}(0)=s\left(s^{i}(0)\right)$. 
and according to property 2 the set of $k$-winning positions of game 1 is the set of solutions in $x$ of the constraint winning ${ }_{k}(x)$ defined in (3).

For example, with $k=1$ the constraint winning $_{k}(x)$ is equivalent to

$$
x=s(0) \vee x=s(s(0))
$$

and with $k=2$ to

$$
x=s(0) \vee x=s(s(0)) \vee x=s(s(s(s(0)))) \vee x=s(s(s(s(s(0)))))
$$

\subsection{Formalizing game 2 in the algebra of infinite trees}

We also reconsider game 2 introduced in section 2.1. As domain $\mathbf{D}$ we take the set $\mathbf{A}$ of trees constructed on a set $F$ of functional symbols including among others the symbols $0, f, g, c$, of respective arities $0,1,1,2$. We code the vertices $(i, j)$ of the game graph by the trees $c(\bar{i}, \bar{j})$ with $\bar{i}=(f g)^{\frac{i}{2}}(0)$ if $i$ is even, and $\bar{i}=g(\overline{i-1})$ if $i$ is odd ${ }^{4}$. Let $G=(V, E)$ be the graph obtained this way.

The perspicacious reader will convince himself that, as generalized relation move, we can take in the algebra of infinite trees:

$$
\operatorname{move}(x, y) \stackrel{\text { def }}{=} \operatorname{transition}(x, y) \vee(\neg(\exists u \exists v x=c(u, v)) \wedge x=y)
$$

with

$$
\begin{aligned}
& \operatorname{transition}(x, y) \stackrel{\text { def }}{=}\left[\begin{array}{l}
\exists u \exists v \exists w \\
{\left[\begin{array}{l}
(x=c(u, v) \wedge y=c(u, w)) \vee \\
(x=c(v, u) \wedge y=c(w, u))
\end{array}\right]} \\
\hat{1} \\
{\left[\begin{array}{l}
(\exists i u=g(i) \wedge \operatorname{succ}(v, w)) \vee \\
(\neg(\exists i u=g(i)) \wedge \operatorname{pred}(v, w))
\end{array}\right]}
\end{array}\right] \\
& \operatorname{succ}(v, w) \quad \stackrel{\text { def }}{=}\left[\begin{array}{l}
((\exists j v=g(j)) \wedge w=f(v)) \vee \\
(\neg(\exists j v=g(j)) \wedge w=g(v))
\end{array}\right] \\
& \operatorname{pred}(v, w) \stackrel{\text { def }}{=}\left[\begin{array}{l}
\left(\exists j v=f(j) \wedge\left[\begin{array}{l}
(\exists k j=g(k) \wedge w=j) \vee \\
(\neg(\exists k j=g(k)) \wedge w=v)
\end{array}\right]\right) \vee \\
\left(\exists j v=g(j) \wedge\left[\begin{array}{l}
(\exists k j=g(k) \wedge w=v) \vee \\
(\neg(\exists k j=g(k)) \wedge w=j)
\end{array}\right]\right) \vee \\
(\neg(\exists j v=f(j)) \wedge \neg(\exists j v=g(j)) \wedge \neg(v=0) \wedge w=v)
\end{array}\right]
\end{aligned}
$$

According to property 2 , the set of $k$-winning positions of game 2 is the set of solutions in $x$ of the constraint winning $g_{k}(x)$ defined in (3).

For example, with $k=1$ the constraint winning $_{k}(x)$ is equivalent to

$$
x=c(g(0), 0) \vee x=c(0, g(0)))
$$

and with $k=2$ to

$$
\left[\begin{array}{l}
x=c(0, g(0)) \vee x=c(g(0), 0) \vee x=c(0, g(f(g(0)))) \vee \\
x=c(g(0), f(g(0))) \vee x=c(f(g(0)), g(0)) \vee x=c(g(f(g(0))), 0)
\end{array}\right]
$$

$\overline{{ }^{4} \text { Of course, }(}(f g)^{0}(x)=x$ and $(f g)^{i+1}(x)=(f g)^{i}(f(g(x)))$. 


\section{Quasi-universality of tree constraints}

After all these quantifiers, we move to constraints, which are so expressive that their solving becomes quasi-undecidable.

\subsection{Defining a huge finite tree by a constraint}

We set $\alpha(k)=2^{2}{ }^{2}$, with $k$ occurrences of 2 . More precisely we take

$$
\alpha(0)=1, \quad \alpha(k+1)=2^{\alpha(k)},
$$

with $k \geq 0$. The function $\alpha$ increases in a stunning way, since $\alpha(0)=1, \alpha(1)=2$, $\alpha(2)=4, \alpha(3)=16, \alpha(4)=65536$ and $\alpha(5)=2^{65536}$. Thus $\alpha(5)$ is greater than $10^{20000}$, a number probably much greater than the number of atoms of the universe or the number of nanoseconds which elapsed since its creation!

We suppose that the set $\mathbf{A}$ of trees is constructed on a set $F$ of functional symbols including among others the symbols $0,1,2,3, s, f$, of respective arities $0,0,0,0,1,4$. For $k \geq 0$ let us introduce the constraint:

$$
\text { huge }_{k}(x) \stackrel{\text { def }}{=} \exists z \text { triangle }_{k}(3, x, z, 0)
$$

with still for $k \geq 0$,

$$
\begin{aligned}
& \text { triangle }_{0}(t, x, z, y) \stackrel{\text { def }}{=} z=x \wedge z=y
\end{aligned}
$$

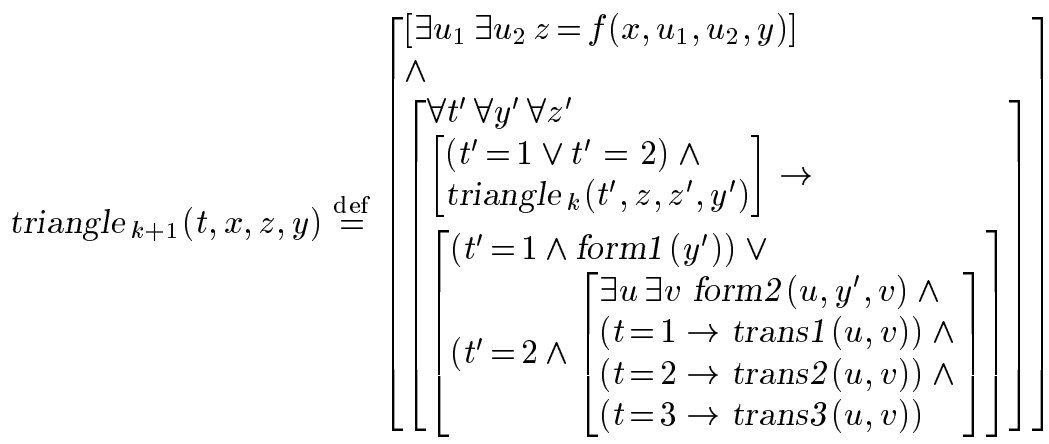

and

$$
\begin{aligned}
& \operatorname{form} 1(x) \quad \stackrel{\text { def }}{=} \exists u_{1} \ldots \exists u_{4} x=f\left(u_{1}, f\left(u_{2}, u_{2}, u_{2}, u_{2}\right), f\left(u_{3}, u_{3}, u_{3}, u_{3}\right), u_{4}\right) \\
& \operatorname{form} 2(x, z, y) \stackrel{\text { def }}{=} \exists u_{1} \ldots \exists u_{6} z=f\left(u_{1}, f\left(u_{1}, u_{2}, u_{3}, x\right), f\left(y, u_{4}, u_{5}, u_{6}\right), u_{6}\right) \\
& \operatorname{trans} 1(x, y) \stackrel{\text { def }}{=} \exists u_{1} \ldots \exists u_{4} x=f\left(u_{1}, u_{2}, u_{3}, u_{4}\right) \wedge\left(y=u_{2} \vee y=u_{3}\right) \\
& \operatorname{trans} 2(x, y) \stackrel{\text { def }}{=} \operatorname{trans} 1(x, y) \vee x=y \\
& \operatorname{trans} 3(x, y) \stackrel{\text { def }}{=} x=s(y)
\end{aligned}
$$


To give a feeling of what triangle ${ }_{k}(t, x, z, y)$ means, here are three trees $x, z, y$ such that triangle $2(t, x, z, y)$ with $t=1, t=2$ and $t=3$, from left to right:
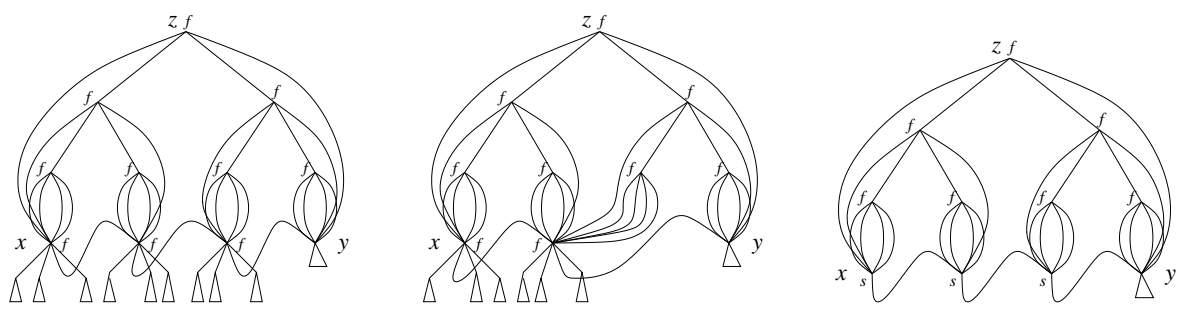

Let us agree that the size $|p|$ of a constraint $p$, is the number of occurrences of all symbols except parentheses and commas. (Constraints could be written in infix notation.) We then have the double property:

Property 3 (small constraint, big tree)

$$
\left|\operatorname{huge}_{k}(x)\right|=9+158 k \quad \text { and } \quad \text { huge }_{k}(x) \leftrightarrow x=s^{\alpha(k)-1}(0) .
$$

To prove the equality, it is sufficient to count:

$$
\begin{aligned}
\left|\operatorname{huge}_{k}(x)\right| & =\mid \text { triangle }_{k}(t, x, z, y) \mid+2, \\
\mid \text { triangle }_{0}(t, x, z, y) \mid & =7, \\
\mid \text { triangle }_{k+1}(t, x, z, y) \mid & =\mid \text { triangle }_{k}(t, x, z, y) \mid+(54+27+23+27+23+4)
\end{aligned}
$$

and to conclude. The proof of the equivalence (in the algebra of infinite trees) is the subject of next subsection.

\subsection{Proof of the second part of property 3}

We write $x\left\{f, k_{1}, \ldots, k_{m}\right\} y$ for expressing that $x$ is a tree whose initial node is labeled $f$ and that there exists $i \in\left\{k_{1}, \ldots, k_{m}\right\}$ such that tree $y$ is the $i$ th daughter of $x$. We also agree that:

$$
\begin{aligned}
& x\left\{f, k_{1}, \ldots, k_{m}\right\}^{0} y \quad \leftrightarrow x=y, \\
& x\left\{f, k_{1}, \ldots, k_{m}\right\}^{n+1} y \leftrightarrow \exists u x\left\{f, k_{1}, \ldots, k_{m}\right\} u \wedge u\left\{f, k_{1}, \ldots, k_{m}\right\}^{n} y
\end{aligned}
$$

with $n \geq 0$.

Given the definition of huge ${ }_{k}(x)$, to show the second part of property 3 it is sufficient to show that, in the algebra of infinite trees, the last of the three following equivalences holds:

$$
\begin{aligned}
& \left(\exists z \text { triangle }_{k}(1, x, z, y)\right) \leftrightarrow x\{f, 2,3\}^{\alpha(k)-1} y \\
& \left(\exists z \text { triangle }_{k}(2, x, z, y)\right) \leftrightarrow \bigvee_{i=0}^{\alpha(k)-1} x\{f, 2,3\}^{i} y \\
& \left(\exists z \text { triangle }_{k}(3, x, z, y)\right) \leftrightarrow x\{s, 1\}^{\alpha(k)-1} y
\end{aligned}
$$


Let us show by induction on $k$ that the three equivalences hold. They hold for $k=0$. Let us assume that they hold for a certain $k \geq 0$ and let us proof that they hold for $k+1$. Definition (4) can be reformulated as

$$
\begin{aligned}
& \text { triangle }_{k+1}(t, x, z, y) \leftrightarrow
\end{aligned}
$$

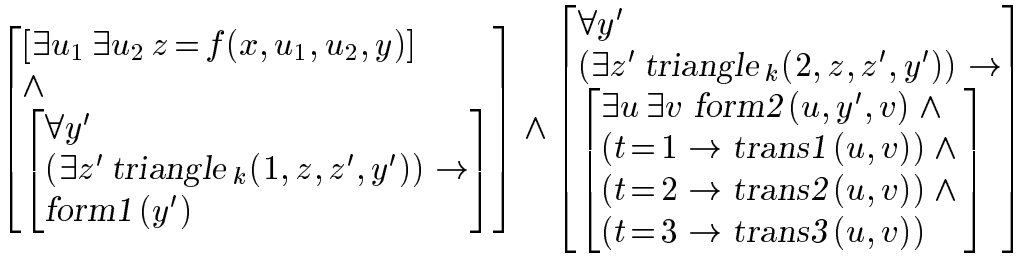

Taking into account our assumptions and using our new notations, we get

$$
\begin{aligned}
& \text { triangle }_{k+1}(t, x, z, y) \leftrightarrow \\
& {\left[\begin{array}{l}
{[z\{f, 1\} x \wedge z\{f, 4\} y]} \\
\wedge \\
{\left[\begin{array}{l}
\forall y^{\prime} \\
z\{f, 2,3\}^{\alpha(k)-1} y^{\prime} \rightarrow \\
\text { form1 }\left(y^{\prime}\right)
\end{array}\right]}
\end{array}\right] \wedge\left[\begin{array}{l}
\forall y^{\prime} \\
{\left[\bigvee_{i=0}^{\alpha(k)-1} z\{f, 2,3\}^{i} y^{\prime}\right] \rightarrow} \\
{\left[\begin{array}{l}
\exists u \exists \text { form } 2\left(u, y^{\prime}, v\right) \wedge \\
(t=1 \rightarrow u\{f, 2,3\} v) \wedge \\
(t=2 \rightarrow u\{f, 2,3\} v \vee u=v) \wedge \\
(t=3 \rightarrow u\{s, 1\} v)
\end{array}\right]}
\end{array}\right]}
\end{aligned}
$$

Since the top of a tree $x$ satisfying form $1(x)$ and the top of a tree $z$ satisfying form $2(x, z, y)$ are respectively of the form
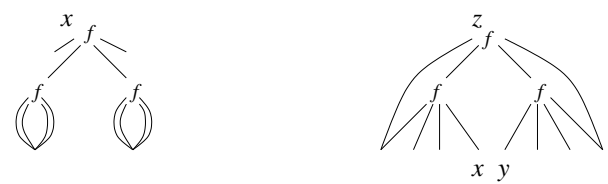

the top of a tree $z$ satisfying triangle $(t, x, z, y)$ is of the form

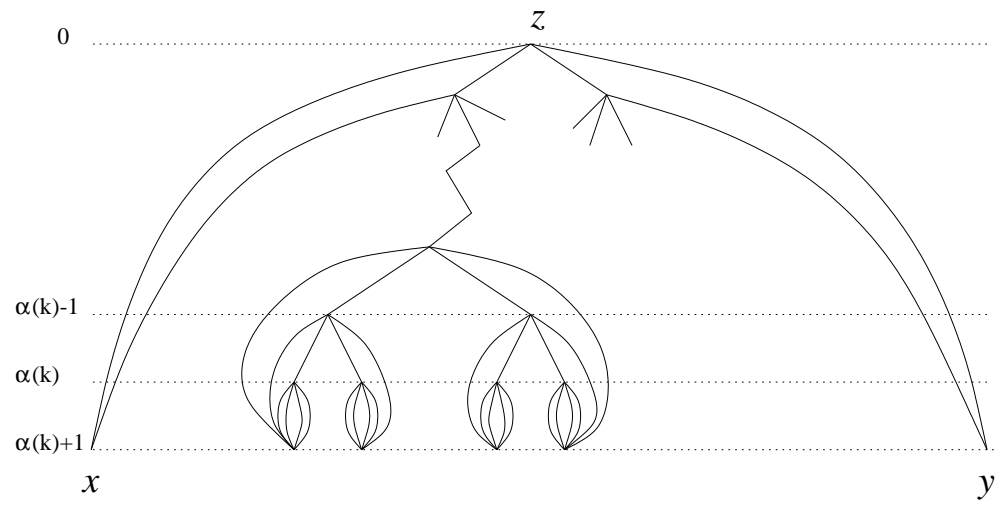


It follows that

$\exists z$ triangle ${ }_{k+1}(t, x, z, y) \leftrightarrow$

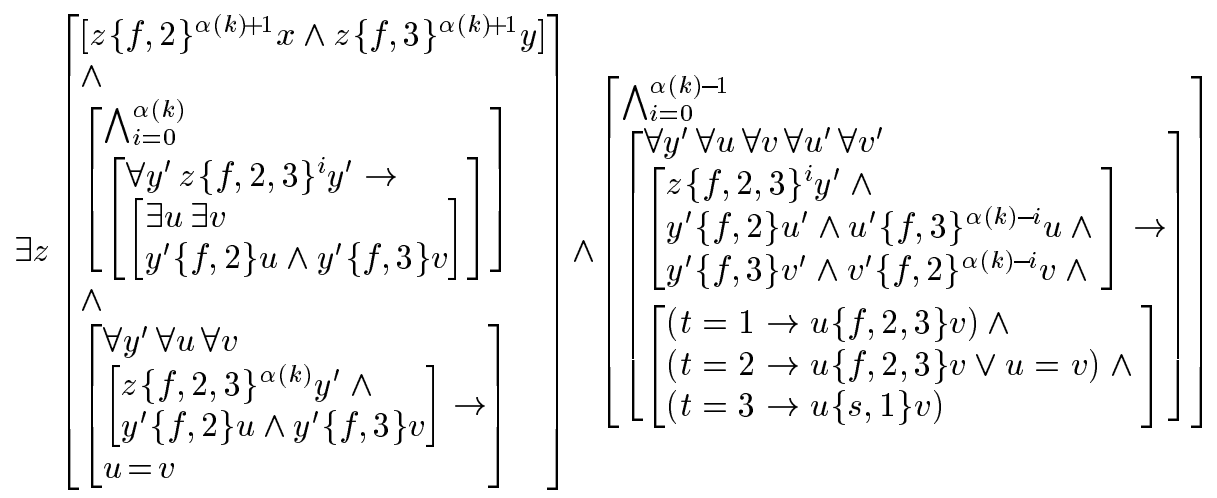

Since, in a binary tree the number of nodes of depth $n$ is equal to $2^{n}$,

$$
\begin{aligned}
& \exists z \text { triangle }_{k+1}(t, x, y, z) \leftrightarrow \\
& \exists u_{1} \ldots \exists u_{\alpha(k)}\left[\left[\begin{array}{l}
x=u_{1} \wedge u_{\alpha(k+1)=y \wedge} \\
\left.\bigwedge_{i=1}^{\alpha(k+1)-1}\left[\begin{array}{l}
\left(t=1 \rightarrow u_{i}\{f, 2,3\} u_{i+1}\right) \wedge \\
\left(t=2 \rightarrow u_{i}\{f, 2,3\} u_{i+1} \vee u_{i}=u_{i+1}\right) \wedge \\
\left(t=3 \rightarrow u_{i}\{s, 1\} u_{i+1}\right)
\end{array}\right]\right]
\end{array}\right.\right.
\end{aligned}
$$

We conclude that the equivalences (5) hold for $k+1$, which ends the proof.

\subsection{Expressing a logic program performing a multiplication}

Let $\operatorname{step}(x, y)$ be a formula involving two free variables $x$ and $y$. If we modify formula triangle $_{k}(t, x, z, y)$ by setting

$$
\operatorname{trans} 3(x, y) \stackrel{\text { def }}{=} x=y \vee \operatorname{step}(x, y)
$$

and if we introduce the formula

$$
\text { iteration }_{k}(x, y) \stackrel{\text { def }}{=} \exists z \exists u \text { triangle }_{k}(3, x, z, u) \wedge \operatorname{trans} 3(u, y)
$$

we then have

$$
\text { iteration }_{k}(x, y) \leftrightarrow \bigvee_{n=0}^{\alpha(k)}\left(\exists u_{0} \ldots \exists u_{n} x=u_{0} \wedge u_{n}=y \wedge \bigwedge_{i=1}^{n} \operatorname{step}\left(u_{i-1}, u_{i}\right)\right)
$$

The binary relation defined by iteration is in some way a bounded transitive closure of the relation defined by step.

Let $T$ be the theory of trees, that is a set of first order propositions which entails all the properties of the algebra of infinite trees which can be expressed as first order propositions. According to logic programming, the formula

$$
\text { times }\left(s^{i}(0), s^{j}(0), x\right)
$$


in the theory

$$
T \cup\left\{\begin{array}{ll}
\forall i \forall j \forall k \forall k^{\prime} & \wedge \\
(\operatorname{times}(0, j, 0) \leftarrow \text { true }) & \wedge \\
\left(\operatorname{times}\left(s(i), j, k^{\prime}\right) \leftarrow \operatorname{times}(i, j, k) \wedge \operatorname{plus}\left(j, k, k^{\prime}\right)\right) & \wedge \\
(\operatorname{plus}(0, j, j) \leftarrow \text { true }) & \hat{\operatorname{plus}}(i, j, k))
\end{array}\right\}
$$

is equivalent to

$$
x=s^{i \times j}(0) .
$$

Given the way a Prolog interpreter works and given equivalence (6), the constraint

$$
\text { iteration }_{k}\left(c\left(f\left(s^{i}(0), s^{j}(0), x\right), 0\right), 0\right)
$$

with

$$
\operatorname{step}(x, y) \stackrel{\text { def }}{=}\left[\begin{array}{ll}
\exists i \exists j \exists k \exists k^{\prime} \exists l & \vee \\
(x=c(f(0, j, 0), l) \wedge y=l) & \left.\vee c\left(f\left(s(i), j, k^{\prime}\right), l\right) \wedge y=c\left(f(i, j, k), c\left(p\left(j, k, k^{\prime}\right), l\right)\right)\right) \\
(x=c & \vee \\
(x=c(p(0, j, j), l) \wedge y=l) & \vee
\end{array}\right]
$$

is equivalent in the algebra of infinite trees to

$$
x=s^{i \times j}(0)
$$

provided that $i(j+2)+1 \leq \alpha(k)$. For $k=5$ we can consider that this restriction is quasi-satisfied. Thus we have a systematic way to replace a logic Horn clauses program by a tree constraint.

\subsection{Universality versus complexity}

Instead of a Prolog machine we can take a Turing machine $M$, and express by step $(x, y)$ the fact that $M$ may move from configuration $x$ to configuration $y$ by executing one instruction. We then conclude that:

Property 4 The result produced by a Turing machine, executing at most $\alpha(k)$ instructions, can be expressed by a tree constraint of size less or equal to a number proportional to $k$.

Here also, by taking $k=5$ it is possible to express any result that the most powerful computer could compute. Thus the tree constraints have a quasi-universal expressiveness and the complexity of the algorithms for solving them must be very high. Let us examine this point in more details and in the case of constraints without free variables.

Let us consider an algorithm as a Turing machine $M$ whose execution terminates for all word $x \in V^{\star}$ given as input. The complexity of $M$ is the mapping of type $\mathbf{N} \rightarrow \mathbf{N}:$

$$
n \mapsto \max \left\{\begin{array}{l|l}
i \in \mathbf{N} & \begin{array}{l}
\text { there exists } x \in V^{\star}, \text { with }|x|=n, \text { such that } M \\
\text { executes } i \text { instructions, with } x \text { as input. }
\end{array}
\end{array}\right\}
$$


Let $\Phi_{\alpha}$ be a set of non-decreasing functions of type $\mathbf{N} \rightarrow \mathbf{N}$ such that

1. the functions of the form $n \mapsto a n+f(b n)$, with $a \in \mathbf{N}, b \in \mathbf{N}$ and $f \in \Phi_{\alpha}$, belong also to $\Phi_{\alpha}$,

2. there exists a language $L$, recognizable by a Turing machine of complexity bounded above by $\alpha$, but by no Turing machine of complexity bounded above by an element of $\Phi_{\alpha}$.

Property 5 Let $T$ be a Turing machine deciding whether a tree constraint without free variables holds. The complexity of $T$ can not be bounded above by an element of $\Phi_{\alpha}$.

Proof. Let us suppose that there exists such a machine $T$ with a complexity bounded above by an element $f$ of $\Phi_{\alpha}$ and let us show that this leads us to a contradiction. Since $\Phi_{\alpha}$ is not empty, the language $L \subseteq V^{\star}$ in part 2 of the definition of $\Phi_{\alpha}$, exists. According to property 4 , to each word $x \in V^{\star}$, corresponds a tree constraints $p_{x}$, without free variables, such that

1. $x \in L$ if and only if $p_{x}$ holds,

2. $\left|p_{x}\right| \leq b|x|$, for some constant $b \in \mathbf{N}$,

3. the transformation $x \mapsto p_{x}$ can be performed by a Turing machine $S$ with a complexity bounded above by $n \mapsto a n$, for some constant $a \in \mathbf{N}$. (This point could be more detailed.)

By linking together the executions of machines $S$ and $T$, we then build a machine $M^{\prime}$ which recognizes $L$ and whose complexity is bounded above by $n \mapsto$ an + $f(b n)$, a function which by definition belongs to $\Phi_{\alpha}$. Thus there is a contradiction about the properties of $L$, which ends the proof.

Under the condition of having shown that, as set $\Phi_{\alpha}$, we can take the set of functions, of type $\mathbf{N} \rightarrow \mathbf{N}$, obtained by finite composition of the elementary functions: $n \mapsto$ cst,,$+ \times, n \mapsto 2^{n}$, we rediscover the result of Sergei Vorobyov [14], but in the spirit of Pawel Mielniczuk [13]:

Property 6 The complexity of an algorithm, which decides whether a tree constraint, without free variables, holds, can not be bounded above by a function obtained by finite composition of elementary functions mentioned above.

\section{Discussions and conclusion}

The presented examples show the contribution of embedded quantifiers and operators $\neg, \wedge, \vee, \rightarrow$ in the expressiveness of tree constraints. They do not really use the fact that the trees may be infinite and are also valid in the algebra of finite trees. It would be interesting to give examples involving infinite trees for coding cyclic structures like finite states automata, context-free grammars or $\lambda$-expressions, as it has been done in $[3,7]$ in the frame of logic programming.

At subsection 3.4 we have provided a glimpse of the huge theoretical complexity of an algorithm for solving tree constraints. However, we have succeeded 
in producing benchmarks on all our examples [10]. The results are summarized in the following table, with CPU times given in milliseconds:

\begin{tabular}{|c||c|c|c|c|}
\hline$k$ & $\begin{array}{c}\text { winning }_{k} \\
\text { game 1 }\end{array}$ & $\begin{array}{c}\text { winning }_{k} \\
\text { game 2 }\end{array}$ & huge $_{k}$ & $\begin{array}{c}\text { iteration }_{k} \\
1 \times 1\end{array}$ \\
\hline 0 & 0 & 0 & 0 & - \\
\hline 1 & 0 & 150 & 0 & - \\
\hline 2 & 10 & 360 & 10 & 70 \\
\hline 3 & 10 & 610 & 230 & - \\
\hline 4 & 20 & 840 & - & - \\
\hline 5 & 30 & 1180 & - & - \\
\hline 10 & 300 & 5970 & - & - \\
\hline 20 & 4270 & 236350 & - & - \\
\hline 40 & 89870 & - & - & - \\
\hline 80 & 3841220 & - & - & - \\
\hline
\end{tabular}

The algorithm is programmed in $\mathrm{C}++$ and the benchmarks are performed on a $350 \mathrm{Mhz}$ Pentium II processor, with $512 \mathrm{Mb}$ of RAM.

It must be noted that we were able to compute the $k$-winning positions of game 1 with $k=80$, which corresponds to a formula involving an alternated embedding of more than 160 quantifiers. We were prepared to experience difficulties in computing the tree of $\alpha(k)$ nodes, beyond $k=3$, since $\alpha(4)$ is already 65536. With respect to multiplication by iteration ${ }_{k}$, we were unable to succeed beyond $k=2$ and had to satisfy ourselves with the computation of $1 \times 1$ !

These test have also removed some of our doubts about the correctness of the complicated formulae of our examples, even if, for readability, we have introduced predicates for naming sub-formulae. Of course the definitions of theses predicates are supposed not to be circular and the solver unfold and eliminates them in a first step.

If circular definitions are accepted then our constraints look like generalized completions of logic programs [2]. Our solver can also take into account such possibly circular definitions by delaying their unfoldings as much as possible. With bad luck the solver does not terminate, with luck it terminates and generates obligatory a simplified constraint without intermediary predicates.

\section{References}

1. Benhamou F., P. Bouvier, A. Colmerauer, H. Garetta, B. Giletta, J.L. Massat, G.A. Narboni, S. N'Dong, R. Pasero, J.F. Pique, Touraïvane, M. Van Caneghem and E. Vétillard, Le manuel de Prolog IV. PrologIA, Marseille, June 1996.

2. Clark K.L., Negation as failure, in Logic and Databases, edited by H. Gallaire and J. Minker, Plenum Press, New York, pp. 293-322, 1978.

3. Colmerauer A., Prolog and Infinite Trees, in Logic Programming, K.L. Clark and S.A.. Tarnlund editors, Academic Press, New York, pp. 231-251, 1982.

4. Colmerauer A., Henry Kanoui andt Michel Van Caneghem, Prolog, theoretical principles and current trends, in Technology and Science of Informatics, North 
Oxford Academic, vol. 2, no 4, August 1983. English version of the journal TSI, AFCET-Bordas, where the paper appears under the title: Prolog, bases théoriques et développements actuels.

5. Colmerauer A., Equations and Inequations on Finite and Infinite Trees, in Proceeding of the International Conference on Fifth Generation Computer Systems (FCGS-84), ICOT, Tokyo, pp. 85-99, 1984.

6. Colmerauer A., An Introduction to Prolog III, Communications of the ACM, 33(7) : 68-90, 1990.

7. Coupet-Grimal S. and O. Ridoux, On the use of advanced logic programming features in computational linguistics. The Journal of Logic Programming, 24(12), pages $121-159$.

8. Courcelle B., Fundamental Properties of Infinite Trees, Theoretical Computer Science, 25(2), pp. 95-169, March 1983.

9. Courcelle B., Equivalences and Transformations of Regular Systems - Applications to Program Schemes and Grammars, Theoretical Computer Science, 42, pp. 1$122,1986$.

10. Dao T.B.H., Résolution de contraintes du premier ordre dans la théorie des arbres fini ou infinis, Journées Francophones de Programmation Logique et Programmation par Contraintes (JFPLC'2000), Marseille, June 2000, proceedings to be published by Hermes Science Publications.

11. Huet G., Résolution d'équations dans les langages d'ordre $1,2, \ldots, \omega$. ., Thèse d'Etat, Université Paris 7, 1976.

12. Maher M.J., Complete Axiomatization of the Algebra of Finite, Rational and Infinite Trees, Technical report, IBM - T.J.Watson Research Center, 1988.

13. Mielniczuk P., Basic Theory of Feature Trees, submitted to Journal of Symbolic Computation, also available at http://www.tcs.uni.wroc.pl/ ${ }^{\text {mielni. }}$

14. Vorobyov S., An Improved Lower Bound for the Elementary Theories of Trees, Proceeding of the 13th International Conference on Automated Deduction (CADE'96). Springer Lecture Notes in Artificial Intelligence, vol 1104, pp. 275287, New Brunswick, NJ, July/August, 1996. 